\title{
Immunotherapy biomarkers 2016: overcoming the barriers
}

\author{
James L. Gulley', Jay A. Berzofsky², Marcus O. Butler ${ }^{3}$, Alessandra Cesano ${ }^{4}$, Bernard A. Fox ${ }^{5}$, Sacha Gnjatic ${ }^{6}$ \\ Sylvia Janetzki ${ }^{7}$, Shyam Kalavar ${ }^{8}$, Vaios Karanikas ${ }^{9}$, Samir N. Khleif ${ }^{10}$, Ilan Kirsch ${ }^{11}$, Peter P. Lee ${ }^{12}$, Cristina Maccalli ${ }^{13}$, \\ Holden Maecker ${ }^{14}$, Jeffrey Schlom ${ }^{15}$, Barbara Seliger ${ }^{16}$, Janet Siebert ${ }^{17}$, David F. Stroncek ${ }^{18}$, Magdalena Thurin ${ }^{19}$, \\ Jianda Yuan ${ }^{20}$ and Lisa H. Butterfield ${ }^{21^{*}}$
}

\begin{abstract}
This report summarizes the symposium, 'Immunotherapy Biomarkers 2016: Overcoming the Barriers', which was held on April 1, 2016 at the National Institutes of Health in Bethesda, Maryland. The symposium, cosponsored by the Society for Immunotherapy of Cancer (SITC) and the National Cancer Institute (NCI), focused on emerging immunotherapy biomarkers, new technologies, current hurdles to further progress, and recommendations for advancing the field of biomarker development.
\end{abstract}

Keywords: Biomarkers, Immunotherapy, Immune monitoring, High throughput, Baseline measures, Tumor microenvironment

\section{Introduction}

Dr. James L. Gulley and Dr. Lisa H. Butterfield opened the meeting with an introduction. The field of cancer immunotherapy has enjoyed unprecedented successes due to positive and durable clinical trial outcomes, the development of new drugs, biologics and combination therapies, and the approval of immunotherapeutics such as immune checkpoint inhibitors. Despite this progress, the current patient response rates and toxicities associated with immunotherapies have created a sense of urgency to determine which patients would most benefit from the treatment option. The identification of immune biomarkers will help to fill knowledge gaps by providing valuable predictive and prognostic information, and insights on the mechanisms that underlie patient responses or resistance to immunotherapy. This would allow development of treatment plans specific to each patient, which could help avoid selection of ineffective therapies, toxicities, and the resulting need to treat these toxicities. Furthermore, the rational design of combination therapies only becomes possible once mechanisms of action and resistance are fully elucidated.

\footnotetext{
* Correspondence: butterfieldl@upmc.edu

${ }^{21}$ Department of Medicine, Surgery and Immunology, University of

Pittsburgh Cancer Institute, 5117 Centre Avenue, Pittsburgh, PA 15213, USA

Full list of author information is available at the end of the article
}

Among current hurdles to the identification and development of clinically relevant biomarkers is the lack of standardized conditions for the collection and storage of specimens. Indeed, the data obtained from variably banked specimens are inconsistent, and at present, many trials bank only non-viable tumor and/or serum samples. The costs associated with immune assays can also be prohibitive, as robust and reproducible signals cannot be obtained by testing small numbers of samples, and attempts to keep costs low by focusing on one or two 'best guess' assays could lead investigators to miss the most accurate biomarker.

The availability of novel technologies and high throughput approaches (e.g. mass cytometry, whole exome sequencing, gene expression profiling, TCR diversity, epigenetics, etc.) offers unique opportunities and challenges for the field of biomarker development. Through the use of these powerful techniques, a multitude of questions can be addressed with a single sample, yet the resulting quantity and complexity of data require unique analytical considerations. The field of immune monitoring is also influenced by the discovery that metabolic considerations, the microbiome, and signaling pathway modulation all affect the immune system. Indeed, many standard of care therapies are now known to operate through immune mechanisms. 
The SITC Biomarkers Task Force was convened to address the current state of the field, and recommend avenues for progress. The 'Immunotherapy Biomarkers 2016: Overcoming the Barriers' symposium focused on outcomes of topics addressed by four Task Force Working Groups. Divided between working groups (WGs), the following themes were discussed: Immune monitoring, assay standardization and validation (WG1); New developments in biomarker assays and technologies (WG2); Systematic assessment of immune regulation and modulation (high throughput approaches) (WG3); and Baseline immunity, tumor immune environment and outcome prediction (WG4). This report summarizes the outcomes from the meeting.

\section{Immunologic monitoring, assay standardization and validation (WG1)}

WG1 was introduced by Dr. Magdalena Thurin. Putative biomarkers predictive of response to immunotherapy must undergo high quality and rigorous validation. As a result, very few biomarkers are approved as clinically useful. A major hurdle is that immune modulation affects many cell types and complex interactions while many drugs and small molecule inhibitors target single proteins or pathways. Greater understanding of the complexity of the validation process, especially for markers based on immune response, is needed in order to build up the resources available for immune monitoring. The two volume white papers from WG1, in which the analytical and clinical validation process, and regulatory considerations are discussed in depth, have been published $[1,2]$.

Dr. Alessandra Cesano presented an overview of the challenges faced in translating biological data into predictive biomarkers and described the example of Prosigna as a cleared diagnostic. A combination of factors from the host, cancer cells, and tumor microenvironment (TME), including the microbiome, shape interactions between the tumor and the immune system. This presents various hurdles, the first being the complexity of integrating these interactions with an informative analyte (DNA, RNA, and proteins) to yield a multivariate, multi-analyte assay. Although tens of thousands of markers in the GVK Biosciences (GVK Bio) Online Clinical Biomarker Database (GOBIOM) developed in collaboration with the U.S. Food and Drug Administration (FDA) have been identified, only a fraction of these have been developed into validated genomic biomarkers for FDA-approved drugs, and none have become multiplex, in vitro companion diagnostics. For a predictive biomarker to be applied in the clinic, it must have analytic (the ability to accurately and reliably measure analytes of interest in clinical specimens representative of the target population) and clinical validity, in addition to clinical utility. Multiple organizations have published guidelines for the validation of diagnostic tests, with recommendations regarding analytic sensitivity, specificity, reproducibility, and assay robustness [3, 4]. Dr. Cesano suggested that the co-development of drugs and companion diagnostics could address the biomarker deficiency. Although such an approach would increase the complexity of drug development, it may make the process more successful, and has great potential value for patients and health system resource utilization.

Dr. Ilan Kirsch detailed the capabilities of highthroughput PCR-based sequencing technology for T cell receptor (TCR) clonality assessment. The basis of this technology arose from the fact that the genes that encode functional immune receptors initially exist in germline DNA as a number of discrete, non-contiguous segments (variable, $\mathrm{V}$, diversity, $\mathrm{D}$, and joining, J). As a prerequisite to the formation of a functional immune receptor, a site-specific DNA breakage and rejoining event occurs that unifies any combination of these segments into a contiguous $\mathrm{V}(\mathrm{D}) \mathrm{J}$ region, which encodes the hypervariable part of the immune receptor. The assay uses forward primers from the $\mathrm{V}$ segments, and reverse primers from the J segments, to amplify and then sequence the area of this rearrangement event, thus creating a unique "barcode" of the lymphocyte and all clonal progeny of the rearranged cell. In this way, immunosequencing can quantify and specify every $\mathrm{B}$ and/or T cell in a sample of interest with a high degree of sensitivity and reproducibility. The superior accuracy of immunosequencing has been demonstrated in side-by-side comparisons with multi-parameter flow cytometry, in which immunosequencing successfully detected minimal residual disease in samples from patients with both $\mathrm{T}$ and $\mathrm{B}$ cell hematologic malignancies that were missed by flow cytometric techniques [5]. Additional applications of high-throughput clonality assessment include insights into drug mechanisms, measurements of immune system dynamics, and prognostic potential. In a study using immunosequencing to assess the number and clonality of tumor-infiltrating lymphocytes (TIL) from tissue samples of stage II DNA mismatch repair-proficient colon cancer patients, it was found that patients with belowmedian clonality and TIL number were at higher risk for disease recurrence [6]. This type of TIL assessment also may predict response to therapy. When applied to the setting of melanoma, patient samples with TIL beneath the median number and level of clonality were less likely to respond to anti-PD-1 therapy [7].

Dr. Sylvia Janetzki discussed challenges associated with single cell functional immune assay validation. The inherent variability associated with assays such as ELISPOT and intracellular cytokine staining [8], due to either the assay itself or due to the sample, needs to be considered. Sample quality must be addressed in pre-analytical validation and 
many guidelines already exist regarding peripheral blood mononuclear cell (PBMC) processing [9], avoidance of granulocyte contamination, apoptosis assessment, and overnight resting of samples. Assay variability arises from the lack of standardized protocols as well as the absence of a "gold standard", which makes accuracy impossible to determine. To address the issue of assay variability, participation in proficiency panels [10] is recommended, as they provide an analysis of one's own measurement in relation to measurements from many other groups in the field using many different protocols. Open proficiency panel programs already exist for ELISPOT and multimer panels and are available to all labs on a non-profit basis. In addition to evaluating the relative accuracy of data, proficiency panels can be used to identify critical protocol variables that are predictive of assay performance. These critical variables have been collected and summarized in published harmonization guidelines to be used by labs for optimization of assay outcomes [11], and are now available for ELISPOT assay and analysis, multimer staining, intracellular cytokine staining (ICS) assay, and gating. Finally, as precision is the most important parameter to ensure confidence in data analysis, intra-assay, inter-assay, and interoperator precision need to be demonstrated in the form of repeatable results with a low false positive rate once assays have been harmonized.

Dr. Shyam Kalavar from the U.S. FDA presented on regulatory considerations for in vitro diagnostic devices in cancer immunotherapy. Companion in vitro diagnostic (IVD) devices provide information that is essential for the safe and effective use of a corresponding therapeutic product (e.g. PD-L1 IHC 22C3 pharmDx diagnostic test manufactured by Dako A/S (now Agilent Technologies)). In contrast, complementary diagnostics are tests that identify a biomarker-defined subset of patients that is expected to respond particularly well to a drug, and aid risk/benefit assessments for individual patients, but are not pre-requisites for receiving the drug (i.e., are not companion diagnostics). An example of a complementary IVD device is PD-L1 IHC 28-8, manufactured by Dako A/S (now Agilent Technologies) for non-squamous NSCLC and melanoma. As commercialization of IVD requires FDA clearance, a key regulatory question is whether adequate analytical and clinical performance data are available to be approved contemporaneously with the drug approval. In the case of immunohistochemistry-based assays such as PD-L1 IHC $22 \mathrm{C} 3$, analytical validation studies include biochemical characterization of the antibody, tests of assay sensitivity and specificity, precision, inter-laboratory reproducibility, assay robustness, the impact of pre-analytical variables, stability, and control cell line validation. Additional information regarding IVD regulation is available in guidance documents provided by the FDA to help direct validation of such devices.
Each section of the meeting had time for a panel discussion. The speakers addressed several questions, including those relating to publication of assay details for proper evaluation by the field.

\section{New developments in biomarker assays and new technologies (WG2)}

Dr. Jianda Yuan introduced the WG2 areas of focus. Over the past year, the members of Biomarkers WG2 have collaborated to evaluate new technologies. These include quantitative real-time PCR-assisted cell counting (qPACC) [12], protein microarray ('seromics') [13], flow and mass cytometry [14], multiplexed tissue biomarker imaging [15], and whole exome sequencing for mutation load/ neoantigen discovery [16]. All the resulting papers have been published in the Journal for ImmunoTherapy of Cancer (JITC) technology primer series [17-23], with still more on the horizon. Additionally, the WG2 white paper highlights novel technologies and emerging biomarkers relevant to individualized cancer immunotherapy, with recommendations for best practices unique to each [24].

As the prognostic power of TILs continues to gain widespread recognition $[25,26]$, Dr. Bernard A. Fox believes that multispectral imaging is critical to advancing our understanding of the complex interactions in the TME. Using markers of immune cell subsets, phenotype, and function [27], standardized multispectral immunohistochemistry is ideally suited to address interactions between multiple parameters, especially when patient sample availability is a consideration. However, difficulties associated with this technique include high background fluorescence, availability of antibodies, photobleaching, and image analysis [28]. The development of a tyramide signal amplification reagent was a breakthrough in multispectral technology that obviates such issues. This technique was used to interrogate tumors from a cohort of patients with melanoma to determine that the CD8:FoxP3 ratio predicts ability to generate TIL, the predictive power for which increases with the addition of PD-L1 [29]. The examination of multiple parameters thus offers value compared to single parameter analysis in a complex environment where ratios, relative positions, and functional analyses are mechanistically important. Dr. Fox suggested that advancements in multispectral imaging technology could provide support for stratification of patients for clinical trials in the future.

Dr. Jianda Yuan described how next-generation sequencing technologies such as whole exome sequencing are paving the way for precision oncology. Mutation-derived antigens (neoantigens) have long been an area of research interest, but the patient-specific nature of neoantigens represented a hurdle to the broader application of these data for a therapeutic purpose. The advent of whole exome sequencing has allowed for a comprehensive description of 
the mutation load, termed the "mutational landscape", which has been a valuable resource to the field of immunotherapy, since neoantigen immunogenicity has been shown to be a correlate of response to treatment. For example, neoantigen-specific $T$ cell reactivity was demonstrated via tumor exome sequencing in ipilimumab-responsive melanoma [30], and most patients who responded to ipilimumab treatment had a relatively high mutation load [31]. Similarly, the nonsynonymous mutation burden was associated with clinical benefit of anti-PD-1 therapy in patients with NSCLC [32], and MMR deficiency in colorectal cancer correlated with better clinical response to PD-1 blockade [33]. Potential uses of whole exome sequencing for neoantigen discovery and precision oncology include: prediction of patient response to vaccine and cancer immunotherapy, identification of new targets, therapeutic neoantigen vaccination, adaptive neoantigen $\mathrm{T}$ cell transfer therapy, and the design of effective combination immunotherapies. Strategies to move the field forward include combining approaches that integrate function (e.g. ELISPOT), phenotype (e.g. multicolor flow cytometry), and signature (e.g. whole exome sequencing, gene expression profile).

Dr. Holden Maecker spoke of the need to systematically measure immunocompetence in cancer patients, as traditional radiation and chemotherapy can be immunosuppressive, and immunotherapeutic agents interact directly with the immune system. Such an assessment of circulating immune subsets and functions could be prognostic of an overall response or a response to specific agents [34]. Mass cytometry (CyTOF) is a powerful alternative to fluorescence flow cytometry that can simultaneously assess multiple parameters with little to no spillover between channels. Dr. Maecker described two studies that employed the use of CyTOF technology to interrogate the immune systems of cancer patients at various time points, both of which suggested that specific immune profiles may be prognostic for response to immunotherapy. In particular, the presence of CD8+ central memory $\mathrm{T}$ cells and/or IL-2-producing $\mathrm{T}$ cells were associated with response to treatment. This immune profile may be a common predictor across multiple chronic conditions, as lack of CD8+ T cells that make IL-2 has been associated with disease progression in HIV [35]. The mTOR pathway might represent a means of boosting the central memory compartment in order to improve response rates for immunotherapies, as mTOR inhibition has been shown to reduce PD-1 expression and increase central memory $\mathrm{T}$ cells $[36,37]$.

Multifaceted immunomonitoring techniques to identify biomarkers predictive of clinical outcome were addressed by Dr. Cristina Maccalli. To capture the complexity of tumor-immune cell interactions, a multifaceted approach integrates different techniques for assessing immune cell phenotypes, functional capabilities, and negative immunoregulatory factors within the same patient sample. Such an approach was applied in a multicenter, controlled study of patients with melanoma being treated with an anti-CTLA-4 agent plus chemotherapy [38, 39]. Initially, standardized multiparameter flow cytometry was used for phenotype analysis of peripheral blood mononuclear cells (PBMC) to identify different subsets of T cells, NK cells, and B cells both at baseline and treatment. Soluble serum factors such as secreted natural killer group 2D (NKG2D) receptor ligands, which are upregulated in tumor cells and can impair $\mathrm{T}$ cell responses were also measured. These data were integrated with classification and regression trees (CART) and an interesting finding emerged, in which patients with longer overall survival were lacking the soluble ligand and had an increase in the frequency of CD4+ BTLA+ memory T cells and Th17-like cells at baseline. Further investigation with functional assays revealed a correlation between antigen-specific $\mathrm{T}$ cell responses and clinical outcomes [40]. Thus, multifaceted immunomonitoring platforms can help identify a functional immunological signature predictive of clinical outcome to immunotherapy, although this platform needs to be optimized and validated with a large cohort of patients undergoing immunotherapy treatments. Dr. Maccalli concluded by stating that the contemporaneous analysis of tumor and peripheral blood could define whether i) a specific tumor immunophenotype is characterized by a distinct peripheral blood gene signature and ii) those signatures correlate with clinical outcome. If it proves that gene signatures correlate with a peripheral blood cell subset phenotype, this would allow a detailed characterization of specific immunophenotype associated with patients' clinical outcome.

The WG2 section had a panel discussion with the speakers. Topics discussed included the timing of sample testing, analysis of primary tumors and metastases, and the extent to which technologies and approaches can be prioritized based on existing data.

\section{Assessment of immune regulation and modulation systematically (high throughput approaches) (WG3)}

Dr. David F. Stroncek presented the areas focused on by the third WG. WG3 sought to provide guidance on which tissue sources should be considered for analysis and monitoring during the process of clinical trial design. Sample materials discussed included serum/plasma, peripheral blood leukocytes, and the microbiome (a focus of the WG3 white paper). In addition, the means by which the tissue should be evaluated, such as proteomics, flow cytometry, gene expression, micro RNA expression, and mutation analysis approaches were considered, along with recommendations for data analysis. Adoptive cell therapies were also discussed. The rapid evolution of the science, and of 
analytical methods, as well as the very large quantities of data involved, present challenges to developing state-ofthe-art guidance [41].

Dr. Peter P. Lee focused on three main aspects of the tumor microenvironment: 1) the importance of the tumor microenvironment to clinical outcomes, 2) the equal importance of the number of cells present and the spatial relationships between them, and 3) the tumor-draining lymph nodes (TDLN), which are the major site of cancer cell-immune cell interaction. Tumors comprise more than cancer cells alone, since roughly $50 \%$ of human tumors are $>50 \%$ stroma. Patients with 'stroma-rich' tumors have poorer clinical outcomes because stromal cells provide growth and metabolic factors that protect cancer cells from a variety of anti-cancer drugs, including chemotherapeutic agents and targeted drugs such as BRAF inhibitors. Indeed, stromal cell density appears to be an independent prognostic marker in breast, colorectal, pancreatic, and other cancers. As mentioned by other presenters, intratumoral immune cells are also predictive of clinical outcome, which led to the Immunoscore effort [42] to aid in clinical decision-making. The interplay between cancer cells, immune cells, and non-immune stromal cells within the TME can be studied using an automated quantitative pathology imaging system. This system allows for the identification of spatial immune cell patterns within the tumor, which may have implications for clinical outcomes. For example, to address whether TDLN functionality is altered in cancer, immune cell populations were evaluated in disease-free versus relapsed breast cancer patients. A significantly higher percentage of CD4+ T cells and CD1a + dendritic cells (DC) was found in the draining lymph nodes of patients who remained disease-free versus those who had relapsed, and this immune profile alone correlated with disease-free survival [43]. Spatially, DC within lymph nodes of disease-free patients were found in clusters, whereas the architecture of DC clusters from the nodes of patients whose disease had relapsed were disrupted -a pattern that also correlated with clinical outcome [44]. Quantitative, spatial image analysis of tumors and TDLN will be highly informative in understanding how therapies modulate the balance between cancer and host immune responses.

The importance of monitoring adoptive cellular therapies as a means of improving clinical outcomes for patients was presented by Dr. David F. Stroncek. Multiple studies of tumor-infiltrating lymphocytes (TIL) as sources of autologous cells for transfer in patients with melanoma have yielded markers indicative of clinical outcome. Specifically, higher total numbers and percent of CD8+ T cells infused are associated with improved outcomes, as is persistence of $\mathrm{T}$ cell clones post-infusion. Other markers have been correlated with outcomes in individual studies and may merit additional investigation, such as telomere length and expression of CD27 [45]. Studies of CD19 CAR T cells in patients with lymphoma or acute lymphocytic leukemia (ALL) have demonstrated that peak levels of CAR T cells with a high CD8:CD4 $\mathrm{T}$ cell ratio, and higher numbers of $\mathrm{CD} 8+$ effector $\mathrm{T}$ cells, are associated with a better response [46-48]. Factors that influence high peak blood levels of CD19 CAR T cells include the presence of CD19 + cells at the time of infusion (lymphoma) [47] and quantities of blasts in bone marrow (ALL). Dr. Stroncek also commented on three complications associated with adoptive cellular therapies: tumor lysis syndrome, cytokine release syndrome (CRS), and neurotoxicity, and the use of biomarkers to assess them. Patients with a higher tumor burden and greater numbers of circulating CAR $\mathrm{T}$ cells are more likely to experience CRS [46]. In the clinic, elevated levels of IFN $\gamma$ and IL-6, or C-reactive protein (CRP) as a surrogate for IL-6, can be tested for, as markers of toxicity [46]. Closing recommendations focused on monitoring levels of adoptively transferred cells; analyzing infused cells for phenotype, gene expression, and polymorphisms; and tracking CRP levels post-treatment.

Dr. Barbara Seliger opened the discussion about immune monitoring of clinical trials by noting that the primary goal of cancer immunotherapy is to increase antitumor activity by activating the immune response, altering the tumor microenvironment, and overcoming tumorassociated barriers. In order to analyze the efficacy of these strategies, it is necessary to integrate multiple highthroughput technologies, which requires investigators to obtain patient samples of peripheral blood, tumor cells, and tumor-infiltrating cells. Ideally, samples should be collected prior to, during, and after treatment, depending on the type of immunotherapy used. Although such an approach is best suited for peripheral blood and in the setting of melanoma, the discovery of links between the immune response and the expression of immune modulatory molecules on tumors may present alternative approaches to monitoring. For example, a decrease in tumor antigen expression is associated with a lack of tumorspecific immune response, and alterations in class I antigen presentation can lead to reduced effector cell cytotoxicity. [49]. It is also important to note that certain types of immunotherapy, including peptide vaccination, checkpoint inhibitors, and adoptive $\mathrm{T}$ cell transfer, have been shown to allow for the evolution of acquired resistance to treatment by selecting for HLA class I loss variants. HLA$\mathrm{G}$ is a non-classical HLA class I molecule that is a putative novel inhibitory checkpoint, as it is often over-expressed in tumors, can inhibit NK and T cell mediated cytotoxicity, and is associated with poorer prognoses in a variety of solid tumors [50]. Dr. Seliger closed by saying that it is important to understand the mechanisms of immune escape in order to appropriately apply immunomodulatory agents, but immune monitoring should not cease once a 
therapy has been selected. Rather, continued monitoring is an important means of addressing resistance to treatment that may emerge over time.

Ms. Janet Siebert described the current state of immune monitoring as a compilation of "humpty dumpty data sets" in her presentation on Analysis of the Systemic Host Response. Her statement emphasized that contemporary data sets are generated through interrogation of multiple tissues, using multiple assays, from multiple organizations (from multiple intra- or inter-institutional cores), across academia, industry, and government. Such fragmentation could reduce the feasibility of measuring of the systemic response. Instead, the field needs to move toward the compilation of integrated heterogeneous data sets or "het sets", which use a consistent assay-agnostic format that spans assays, tissues, and organizations, and supports analysis of the systemic cross-compartment response. Advantages of het sets include a common technical and conceptual representation of an otherwise unwieldy data set, the application of the same analytical tools to a large number of analytes from different assays, and the ability to apply established multivariable analytical approaches to the integrated whole. Ideally, these analytical approaches would be well-established and magnitude-insensitive with both visual and quantitative results that can be easily validated in follow-up studies. Decision tree classification is an example of a suitable analytical approach that seeks to determine which analytes most cleanly separate patients into responder and non-responder groups. These can be supplemented with reference data sets to ensure that thresholds are not arbitrary or over-fitted. To address systemic responses that span assays or tissues, linear regression modeling can be used to capture the relationship between analyte pairs [51]. The resulting complex immunological data can then be synthesized with a network arc diagram to provide succinct visualization, and insights into underlying the biological mechanisms of interest, such as responsiveness to therapy [52].

During the WG3 panel discussion, the speakers commented on the use of high throughput technologies to test adoptive cellular therapies, and detection of tertiary lymphoid structures.

\section{Prediction of clinical outcome based on baseline measures (WG4)}

Dr. Sacha Gnjatic introduced the fourth WG and its activities. Aiming to provide recommendations on how to predict a patient's response to treatment through analysis of baseline biomarkers in blood and tumor, including MDSC and other immature myeloid cells, and checkpoint molecule expression. WG4 also focused on the diversity of immune cells present in the TME (including tertiary lymphoid structures), effectiveness of peripheral surrogates of the TME, and new technologies to aid in the comprehensive analysis of baseline immunity (manuscript in revision for the Journal for ImmunoTherapy of Cancer).

Investigations into the tumor microenvironment at a genetic level, as presented by Dr. Samir N. Khleif, seek to address whether genetic changes within the tumor microenvironment can guide the design of cancer immunotherapeutics. The power of this approach was illustrated by investigations into Ras as a mutated antigen immune target. In contrast to prognostic or predictive biomarkers, immune targets are biomarkers that might not correlate strongly with response to treatment, such as PD-L1, but can help direct the development of therapies. Dr. Khleif described a study in which Ras mutations were used as immune target biomarkers. Patients with advanced solid tumors bearing Ras mutations were given a cancer vaccine comprised of autologous peptides along with IL-2, GMCSF, or both. Although most patients developed antigenspecific immune responses, only one patient out of 57 generated productive immunity that went on to eliminate the tumor cells [53]. This disparity led to the discovery that regulatory $\mathrm{T}$ cell (Tregs) are significantly expanded in colon cancer patients with mutated Ras compared to both healthy individuals and colon cancer patients with wildtype Ras. It was found that mutant Ras activates the MEKERK-AP1 pathway to induce secretion of high levels of IL10 and TGF- $\beta 1$, which generate local induction of regulatory $\mathrm{T}$ cells (Treg) in the tumor microenvironment [54]. Ras mutation was noted to be an early event in the development of tumors leading to the proliferation of affected cells, and the resulting induction of Treg serves to support tumor immune escape by creating a suppressive microenvironment that inhibits the anti-tumor immune response. Dr. Khleif suggested that an additional agent targeting Treg could boost the efficacy of a cancer vaccine in patients with Ras mutations. Thus mutated antigens used as immune target biomarkers can also guide investigations into immune bystander effects on the microenvironment, with the potential to inform the development of rational combination treatments.

In a forward-looking discussion, Dr. Vaios Karanikas presented on multiplex immunohistochemistry (IHC) in clinically annotated material: where are we and where are we going? As more clinical data become available from patients treated with cancer immunotherapies, it has become clear that patients with inflamed tumors are more likely to respond to treatment. A deeper understanding of the interactions between the immune system and tumor cells within the tumor microenvironment is needed to achieve the ultimate goal of designing therapeutic strategies capable of inflaming "cold" tumors and addressing immune escape mechanisms. The identification of predictive and prognostic biomarkers using high throughput approaches like multiplex IHC would aid this endeavor and Dr. Karanikas 
offered a number of best practices for consideration. As tumor cells do not develop in isolation, it is imperative to assess the TME comprehensively (immune cells, stroma, and tumor cells), and compare paired samples from the same patient before, during, and after treatment. Choice of technology, e.g., chromogenic vs immunofluorescence, and antibody selection, can be guided by pragmatism. A relevant example of the complex questions that can be addressed by multiplex IHC includes the efforts to define the tumor-infiltrating cell types. This approach demonstrated the prognostic value of specific subsets of immune cell infiltrates $[55,56]$, their spatial distribution within the TME $[57,58]$, and qualitative characteristics such as an exhausted phenotype $[7,59]$. Importantly, the integration of complementary technologies such as multiplex IHC, mutational profiling, and gene expression patterns can overcome the limitations of each method, as well as offer an improved understanding of the TME toward the identification of predictive and prognostic immune biomarkers.

The identification of biomarkers in the peripheral blood - the most readily available tissue for sampling - to assess a patient's response to treatment was the focus of Dr. Marcus O. Butler's Multiplex/Blood Profiles presentation. Central to this approach is a need to identify whether biomarkers in the TME are also present in the peripheral blood. Investigations into circulating PD-1+ lymphocytes, which have been shown to contain $\mathrm{T}$ cells specific for tumor-associated antigens and neoantigens, have provided evidence to suggest that this is possible, although such populations of interest are less frequent in blood [60]. The main tools available to interrogate rare cell populations include flow and mass cytometry and a number of different computational methodologies have been published to guide the analysis of these complex data [61]. In addition to its utility as a window into the TME, the peripheral compartment can be used to try to influence baseline immunity. One such study involved the adoptive transfer of in vitro-activated tumor antigen-specific $\mathrm{T}$ cells into patients with metastatic melanoma, the result of which was a stable increase in anti-tumor central memory cells detectable in the peripheral blood [62]. Upon subsequent treatment with anti-CTLA-4, the transferred cells expanded and generated partial responses [62] that laid the foundation for follow up studies including Adoptive Cell Therapy InVigorated to Augment Tumor Eradication (ACTIVATE): Cohorts 1-3.

In a discussion about $\mathrm{B}$ cells at the tumor site, and systemic humoral responses, as predictive biomarkers, Dr. Sacha Gnjatic noted that B cells organized in tertiary lymphoid structures (TLS) can be identified near tumors and the presence of dense follicular B cells along with mature DC is associated with a good prognosis in non-small cell lung cancer (NSCLC) [63]. Taking these observations a step further, investigators sought to determine whether the specificity of local and circulating antibodies may reflect the immunogenicity of the tumor. Tumor antigen-specific humoral immunity has been previously shown to correlate with $\mathrm{CD} 4+$ and $\mathrm{CD} 8+\mathrm{T}$ cell responses to the cancer-testis antigen NY-ESO-1 $[64,65]$, and more recent studies in patients with NSCLC have gone on to demonstrate correlations between anti-NY-ESO-1 antibody titers and tumor stage, overall survival, and clinical course following antiCTLA-4 immunotherapy [66, 67]. To translate humoral immune monitoring to the clinic, a seromics approach would allow for a sensitive and high-throughput means of detecting alterations in the antibody repertoire as a surrogate for the tumor immune response. Additional work in the area of comprehensive immune monitoring will need to establish appropriate targets that assign functionality to antigen specificity and relate to clinical outcomes.

The WG4 panel discussion addressed issues of tumor acquisition, including cost, logistics, and enabling a culture of routine tissue banking at different institutions and clinical settings.

\section{National cancer institute perspectives on biomarkers}

In a presentation focused on lessons learned from the peripheral immunoscore, Dr. Jeffrey Schlom, discussed applications for the current state of flow-based technology that now allows for identification of 123 immune cell subsets from one tube of blood, using 30 markers. The subsets analyzed include nine standard immune cell subsets and 118 additional subsets relating to maturation and function [68]. In particular, monitoring of multiple peripheral immune subsets can aid in the identification of patients most likely to benefit from immunotherapy. For example, one study of patients with metastatic breast cancer treated with chemotherapy alone or in combination with cancer vaccine sought to use baseline measures of peripheral immunity to discriminate between patients with longer or shorter PFS. Indeed, a correlation between the presence of particular immune cells at baseline and disease progression was noted, although only in the combination therapy arm [69]. Such peripheral immunoscore analyses are intended to complement biopsy analyses, and can be used in many cancer types and stages for which biopsies are not easily obtained. Peripheral immune monitoring can extend beyond cells to the level of antigens. Several studies have demonstrated improved clinical outcomes due to the presence of cascade antigens (epitope spreading) following treatment with cancer vaccines as a result of tumor cell lysis and subsequent cross-priming [70-74]. For example, a trial using the cancer vaccine PROSTVAC in patients with prostate cancer revealed that a majority of patients generated $\mathrm{T}$ cells that mounted an immune response to antigens not present in the vaccine [75]. These data indicate a means by which heterogeneity among 
tumor cells can be minimized therapeutically, and Dr. Schlom suggested that all cancer vaccine clinical trials should include cascade antigen analyses.

Dr. Jay A. Berzofsky discussed the utility of extracellular vesicles (EV) as biomarkers of immune responses and tumor responses in cancer immunotherapy. Although previously underestimated as a homogeneous population, EV are gaining interest as diverse subsets that play many immunoregulatory roles in cancer. Released by both immune cells and tumor cells, EV are $100 \mathrm{~nm}$ particles capable of shuttling "packets" of information in the form of mRNA, miRNA, and protein between cells, the effects of which can be immunostimulatory or immunosuppressive. Similar to standard FACS technology, nanoFACS was developed to analyze, sort, and decode EV subsets based on light scatter and fluorescence. Once sorted, the EV could be further analyzed by molecular profiling via protein expression and RNA/DNA typing. The analysis of EV from patient plasma samples could provide a means of monitoring the immune response and directing treatment decisions, and it has the potential to make both membrane-bound and soluble (exosome-bound) TMEspecific biomarkers accessible via "liquid biopsy".

\section{Conclusions}

In his closing remarks, Dr. James L. Gulley was optimistic that the opportunities presented by the use of biomarkers for immune monitoring outweighed current challenges. Having raised awareness of potential difficulties in immune biomarker research, the SITC Biomarkers Task Force WGs have also made recommendations for how to approach these challenges, and will continue to provide guidance as the field develops.

\footnotetext{
Abbreviations

ALL: Acute lymphoblastic leukemia; CRS: Cytokine release syndrome; CyTOF: Cytometry by time-of-flight, mass cytometry; DC: Dendritic cell(s); EV: Extracellular vesicle(s); FDA: U.S. Food and Drug Administration; ICS: Intracellular cytokine staining; IHC: Immunohistochemistry; IVD: In vitro diagnostic; JITC: Journal for immunotherapy of cancer; NCl: National cancer institute: NKG2D: Natural killer group 2D; NSCLC: Non-small cell lung cancer; PBMC: Peripheral blood mononuclear cells; PFS: Progression-free survival; qPACC: Quantitative real-time PCR assisted cell counting; SITC: Society for immunotherapy of cancer; TCR: T cell receptor; TDLN: Tumor-draining lymph nodes; TIL: Tumor-infiltrating lymphocyte(s); TLS: Tertiary lymphoid structures; TME: Tumor microenvironment; Treg: Regulatory T cell(s); WG: Working group.
}

\section{Acknowledgments}

The views presented in this article do not necessarily reflect those of the U.S Food and Drug Administration. The authors thank Stefanie Haasken, PhD for significant expert editorial assistance and SITC staff for organizing the symposium.

\section{Funding}

Not applicable.

Availability of data and materials Not applicable.

\section{Authors' contributions}

$J G$ wrote and edited the meeting report. JB wrote and edited the meeting report. $\mathrm{MB}$ wrote and edited the meeting report. AC wrote and edited the meeting report. BF wrote and edited the meeting report. SG wrote and edited the meeting report. SJ wrote and edited the meeting report. SK wrote and edited the meeting report. VK wrote and edited the meeting report. SNK wrote and edited the meeting report. IK wrote and edited the meeting report. PL wrote and edited the meeting report. CM wrote and edited the meeting report. HM wrote and edited the meeting report. JS wrote and edited the meeting report. BS wrote and edited the meeting report. JS wrote and edited the meeting report. DS wrote and edited the meeting report. MT wrote and edited the meeting report. JY wrote and edited the meeting report. LB wrote and edited the meeting report. All authors contributed equally to the writing and critical review of this meeting report. All authors read and approved the final manuscript.

\section{Competing interests}

AC is an employee of NanoString Technologies and also has ownership interests in NanoString Technologies; BAF is an employee of UbiVac and holds intellectual property rights and ownership interests in UbiVac, is a consultant for PerkinElmer, Inc. and Argos Therapeutics, Inc., is contracted to do research with Janssen, Bristol-Myers Squibb, Peregrine Pharmaceuticals, Inc., and Viralytics Ltd., has ownership interests in UbiVac, and other relationships with Aduro Biotech, Inc., Advanced Cell Diagnostics, Inc., Definiens, Medlmmune LLC, and Roche; SG holds intellectual property rights and receives royalties from Ludwig Institute for Cancer Research, Ltd., is a consultant for Neon Therapeutics, B4CC, Inc., and Bristol-Myers Squibb, and is contracted to do research for Immune Design and Janssen Pharmaceuticals, Inc;; SJ has ownership interests in Merck, Roche, and Bristol-Myers Squibb.

VK is a full-time employee of Roche and holds intellectual property rights and receives royalties from Roche; SNK is a consultant for Advaxis, Genentech, Inc., AstraZeneca, MedThink Communications, Merus, PDS Biotechnology Corp., Global Health Integrators, Gilead Sciences, Inc., UbiVac, Nektar Therapeutics, Syndax Pharmaceuticals, and NantKwest, serves on advisory boards for Jefferies LLC and Janssen Pharmaceuticals, Inc., is contracted to do research for Advaxis, CureTech Ltd., and PDS Biotechnology Corp., and has ownership interest in Advaxis; IK is an employee of and has ownership interests in Adaptive Biotechnologies; BS has received research funding from Bristol-Meyers Squibb; JS is an employee of CytoAnalytics; JY is an employee of Merck and holds a patent with Adaptive Biotechnologies; LHB is a consultant for Oxford Immunotec, Inc., Affymetrix, Inc., Merck, Biodesix, Inc., Verastem, Inc., and the University of California, Los Angeles, and has ownership interest in Kite Pharma, Inc. All authors declare that they have no other financial or non-financial competing interests.

\section{Consent for publication}

Not applicable.

Ethics approval and consent to participate

Not applicable.

\footnotetext{
Author details

${ }^{1}$ Genitourinary Malignancies Branch, Center for Cancer Research, NCl, 10 Center Dr., 13 N240, Bethesda, MD 20892, USA. Vaccine Branch, Center for Cancer Research, 41 Medlars Dr, Bldg 41 Rm D702D, Bethesda, MD 20892, USA. ${ }^{3}$ Princess Margaret Cancer Center/Ontario Cancer Institute, RM 9-622, 610 University Ave, Toronto, ON, Canada. ${ }^{4}$ NanoString, Inc., 500 Fairview Avenue North, Seattle, WA 98109, USA. ${ }^{5}$ Earle A. Chiles Research Institute, Providence Cancer Center, 4805 NE Glisan Street, Portland, OR 97213, USA. ${ }^{6}$ Department of Hematology/Oncology, Tisch Cancer Institute, Icahn Schoo of Medicine at Mount Sinai, S5-105, 1470 Madison Avenue, Box 1128, New York, NY 10029, USA. 'ZelllNet Consulting, Inc., 555 North Avenue, Fort Lee, NJ 07024, USA. ${ }^{8}$ Center for Devices and Radiological Health, U.S. Food and Drug Administration, 1401 Rockville Pike, Rockville, MD 20852, USA. ${ }^{9}$ Roche Innovation Center Zurich, Wagistrasse 18, Schlieren, Switzerland. ${ }^{10} \mathrm{Georgia}$ Cancer Center, Augusta University, 1120 15th Street, CN-2101A, Augusta, GA 30912, USA. "'Adaptive Biotechnologies, Inc., 1551 Eastlake Ave. E., Seattle, WA 98102, USA. ${ }^{12}$ Department of Immuno-oncology, City of Hope, 1500 East Duarte Road, Duarte, CA 91010, USA. ${ }^{13}$ Department of Translational Medicine, Sidra Medical and Research Center, Doha, Qatar. ${ }^{14}$ Stanford University Medical Center, 299 Campus Drive, Stanford, CA 94303, USA. ${ }^{15}$ National
} 
Cancer Institute, National Institutes of Health, 10 Center Drive, Bldg. 10, Room 8B09, Bethesda, MD 20892, USA. ${ }^{16}$ Institute of Medical Immunology, Martin Luther University Halle-Wittenberg, Magdeburger Str. 2, Halle, Germany. ${ }^{17}$ CytoAnalytics, 3500 South Albion Street, Cherry Hills Village, CO 80113, USA. ${ }^{18}$ Department of Transfusion Medicine, National Institutes of Health, 10 Center Drive, Building 10, Room 3C720, Bethesda, MD 20892, USA. ${ }^{19}$ National Cancer Institute, Cancer Diagnosis Program, DCTD, National Institutes of Health, 9609 Medical Center Drive, Bethesda 20892, MD, USA.

${ }^{20}$ Early Clinical Oncology Development, Merck Research Laboratories, Rahway, NJ 07065, USA. ${ }^{21}$ Department of Medicine, Surgery and Immunology, University of Pittsburgh Cancer Institute, 5117 Centre Avenue, Pittsburgh, PA 15213, USA.

\section{Received: 3 January 2017 Accepted: 13 February 2017} Published online: 21 March 2017

\section{References}

1. Masucci GV, Cesano A, Hawtin R, Janetzki S, Zhang J, Kirsch I, et al. Validation of biomarkers to predict response to immunotherapy in cancer: Volume I - preanalytical and analytical validation. J ImmunoTher Cancer. 2016;4(1):76

2. Dobbin KK, Cesano A, Alvarez J, Hawtin R, Janetzki S, Kirsch I, et al. Validation of biomarkers to predict response to immunotherapy in cancer: Volume II - clinical validation and regulatory considerations. J ImmunoTher Cancer. 2016:4(1):77.

3. Chau CH, Rixe O, McLeod H, Figg WD. Validation of analytic methods for biomarkers used in drug development. Clin Cancer Res. 2008;14(19):5967-76.

4. Lee JW, Weiner RS, Sailstad JM, Bowsher RR, Knuth DW, O'Brien PJ, et al. Method validation and measurement of biomarkers in nonclinical and clinical samples in drug development: a conference report. Pharm Res. 2005;22(4):499-511.

5. Wu D, Emerson RO, Sherwood A, Loh ML, Angiolillo A, Howie B, et al. Detection of minimal residual disease in $B$ lymphoblastic leukemia by highthroughput sequencing of IGH. Clin Cancer Res. 2014;20(17):4540-8.

6. Moreno V, Sanz-Pamplona R, Emerson R, Gruber S, Robins H: Tumor Infiltrating Lymphocytes and Colorectal Cancer Prognosis. In: 15th ASEICA International Congress: 21-23 Oct 2015; Seville, Spain; 2015.

7. Tumeh PC, Harview CL, Yearley JH, Shintaku IP, Taylor EJ, Robert L, et al. PD1 blockade induces responses by inhibiting adaptive immune resistance. Nature. 2014:515(7528):568-71.

8. Janetzki S, Britten CM, Kalos M, Levitsky HI, Maecker HT, Melief CJ, et al "MIATA"-minimal information about T cell assays. Immunity. 2009:31(4):527-8.

9. Mallone R, Mannering SI, Brooks-Worrell BM, Durinovic-Bello I, Cilio CM, Wong FS, et al. Isolation and preservation of peripheral blood mononuclear cells for analysis of islet antigen-reactive $T$ cell responses: position statement of the T-Cell Workshop Committee of the Immunology of Diabetes Society. Clin Exp Immunol. 2011;163(1):33-49.

10. Janetzki S, Price L, Schroeder H, Britten CM, Welters MJ, Hoos A. Guidelines for the automated evaluation of Elispot assays. Nat Protoc. 2015:10(7):1098-115.

11. van der Burg SH, Kalos M, Gouttefangeas C, Janetzki S, Ottensmeier C, Welters MJ, et al. Harmonization of immune biomarker assays for clinical studies. Sci Transl Med. 2011;3(108):108ps44.

12. Kleen TO, Yuan J. Quantitative real-time PCR assisted cell counting (qPACC) for epigenetic - based immune cell quantification in blood and tissue. J Immunother Cancer. 2015;3:46

13. Yuan J, Wang E, Fox BA. Immune Monitoring Technology Primer: protein microarray ('seromics'). J Immunother Cancer. 2016;4:2.

14. Maecker HT, Harari A. Immune monitoring technology primer: flow and mass cytometry. J Immunother Cancer. 2015;3:44.

15. Stack EC, Foukas PG, Lee PP. Multiplexed tissue biomarker imaging. J Immunother Cancer. 2016:4:9.

16. Kvistborg P, Clynes R, Song W, Yuan J. Immune monitoring technology primer: whole exome sequencing for neoantigen discovery and precision oncology. J ImmunoTher Cancer. 2016;4(1):22.

17. Kirsch I. Immune monitoring technology primer: immunosequencing. J Immunother Cancer. 2015;3(1):29.

18. Janetzki S. Immune monitoring technology primer: the enzyme-linked immunospot (Elispot) and Fluorospot assay. J Immunother Cancer. 2015;3(1):30

19. Hawtin RE, Cesano A. Immune monitoring technology primer: Single Cell Network Profiling (SCNP). J ImmunoTher Cancer. 2015;3(1):34.

20. Dobbin KK. Immune monitoring technology primer: clinical validation for predictive markers. J ImmunoTher Cancer. 2015;3(1):40.
21. Brunet LR, LaBrie S, Hagemann T. Immune monitoring technology primer: immunoprofiling of antigen-stimulated blood. J ImmunoTher Cancer. 2016; $4(1): 18$

22. Hermitte F. Biomarkers immune monitoring technology primer: Immunoscore Colon. J ImmunoTher Cancer. 2016;4(1):57.

23. Cesano A. nCounter ${ }^{\circledR}$ PanCancer Immune Profiling Panel (NanoString Technologies, Inc., Seattle, WA). J ImmunoTher Cancer. 2015;3(1):42.

24. Yuan J, Hegde PS, Clynes R, Foukas PG, Harari A, Kleen TO, et al. Novel technologies and emerging biomarkers for personalized cancer immunotherapy. J Immunother Cancer. 2016;4:3.

25. Galon J, Costes A, Sanchez-Cabo F, Kirilovsky A, Mlecnik B, Lagorce-Pages C, et al. Type, density, and location of immune cells within human colorectal tumors predict clinical outcome. Science. 2006;313(5795):1960-4

26. Brambilla E, Le Teuff G, Marguet S, Lantuejoul S, Dunant A, Graziano S, et al. Prognostic Effect of Tumor Lymphocytic Infiltration in Resectable NonSmall-Cell Lung Cancer. J Clin Oncol. 2016;34(11):1223-30.

27. Chen DS, Mellman I. Oncology meets immunology: the cancer-immunity cycle. Immunity. 2013;39(1):1-10.

28. Gerdes MJ, Sood A, Sevinsky C, Pris AD, Zavodszky MI, Ginty F. Emerging understanding of multiscale tumor heterogeneity. Front Oncol. 2014;4:366.

29. Feng Z, Puri S, Moudgil T, Wood W, Hoyt CC, Wang C, et al. Multispectral imaging of formalin-fixed tissue predicts ability to generate tumorinfiltrating lymphocytes from melanoma. J Immunother Cancer. 2015;3:47.

30. van Rooij N, van Buuren MM, Philips D, Velds A, Toebes M, Heemskerk B, et al. Tumor exome analysis reveals neoantigen-specific T-cell reactivity in an ipilimumab-responsive melanoma. J Clin Oncol. 2013;31(32):e439-42.

31. Snyder A, Makarov V, Merghoub T, Yuan J, Zaretsky JM, Desrichard A, et al. Genetic basis for clinical response to CTLA-4 blockade in melanoma. N Engl Med. 2014;371(23):2189-99.

32. Rizvi NA, Hellmann MD, Snyder A, Kvistborg P, Makarov V, Havel JJ, et al. Cancer immunology. Mutational landscape determines sensitivity to PD-1 blockade in non-small cell lung cancer. Science. 2015;348(6230):124-8.

33. Le DT, Uram JN, Wang H, Bartlett BR, Kemberling H, Eyring AD, et al. PD-1 Blockade in Tumors with Mismatch-Repair Deficiency. N Engl J Med. 2015; 372(26):2509-20.

34. Chang S, Kohrt H, Maecker HT. Monitoring the immune competence of cancer patients to predict outcome. Cancer Immunol Immunother. 2014; 63(7):713-9.

35. Nomura LE, Emu B, Hoh R, Haaland P, Deeks SG, Martin JN, et al. IL-2 production correlates with effector cell differentiation in HIV-specific CD8+ T cells. AIDS Res Ther. 2006;3:18.

36. Mannick JB, Del Giudice G, Lattanzi M, Valiante NM, Praestgaard J, Huang B, et al. mTOR inhibition improves immune function in the elderly. Sci Transl Med. 2014;6(268):268ra179.

37. Araki K, Turner AP, Shaffer VO, Gangappa S, Keller SA, Bachmann MF, et al. mTOR regulates memory CD8 T-cell differentiation. Nature. 2009;460(7251):108-12.

38. Di Giacomo AM, Ascierto PA, Pilla L, Santinami M, Ferrucci PF, Giannarelli D, et al. Ipilimumab and fotemustine in patients with advanced melanoma (NIBITM1): an open-label, single-arm phase 2 trial. Lancet Oncol. 2012;13(9):879-86.

39. Di Giacomo AM, Ascierto PA, Queirolo P, Pilla L, Ridolfi R, Santinami M, et al. Three-year follow-up of advanced melanoma patients who received ipilimumab plus fotemustine in the Italian Network for Tumor Biotherapy (NIBIT)-M1 phase II study. Ann Oncol. 2015;26(4):798-803.

40. Maccalli C, Giannarelli D, Capocefalo F, Pilla L, Fonsatti E, Di Giacomo AM, et al. Immunological markers and clinical outcome of advanced melanoma patients receiving ipilimumab plus fotemustine in the NIBIT-M1 study. Oncoimmunology. 2016;5(2):e1071007.

41. Stroncek DF, Butterfield LH, Cannarile MA, Dhodapkar MV, Greten TF, Grivel JC, Kaufman DR, Kong HH, Korangy F, Lee PP, Marincola F, Rutella S, Siebert JC, Trinchieri G, Seliger B. Systematic evaluation of immune regulation and modulation. 2017; doi: 10.1186/s40425-017-0223-8.

42. Galon J, Pages F, Marincola FM, Thurin M, Trinchieri G, Fox BA, et al. The immune score as a new possible approach for the classification of cancer. J Transl Med. 2012;10:1

43. Kohrt HE, Nouri N, Nowels K, Johnson D, Holmes S, Lee PP. Profile of immune cells in axillary lymph nodes predicts disease-free survival in breast cancer. PLoS Med. 2005;2(9):e284

44. Chang AY, Bhattacharya N, Mu J, Setiadi AF, Carcamo-Cavazos V, Lee $\mathrm{GH}$, et al. Spatial organization of dendritic cells within tumor draining lymph nodes impacts clinical outcome in breast cancer patients. J Transl Med. 2013;11:242. 
45. Rosenberg SA, Yang JC, Sherry RM, Kammula US, Hughes MS, Phan GQ, et al. Durable complete responses in heavily pretreated patients with metastatic melanoma using T-cell transfer immunotherapy. Clin Cancer Res. 2011;17(13):4550-7.

46. Lee DW, Kochenderfer JN, Stetler-Stevenson M, Cui YK, Delbrook C, Feldman SA, et al. T cells expressing CD19 chimeric antigen receptors for acute lymphoblastic leukaemia in children and young adults: a phase 1 doseescalation trial. Lancet. 2015:385(9967):517-28.

47. Brudno JN, Somerville RP, Shi V, Rose JJ, Halverson DC, Fowler DH, et al. Allogeneic T Cells That Express an Anti-CD19 Chimeric Antigen Receptor Induce Remissions of B-Cell Malignancies That Progress After Allogeneic Hematopoietic Stem-Cell Transplantation Without Causing Graft-Versus-Host Disease. J Clin Oncol. 2016;34(10):1112-21.

48. Kochenderfer JN, Dudley ME, Kassim SH, Somerville RP, Carpenter RO, Stetler-Stevenson $\mathrm{M}$, et al. Chemotherapy-refractory diffuse large B-cell lymphoma and indolent B-cell malignancies can be effectively treated with autologous T cells expressing an anti-CD19 chimeric antigen receptor. J Clin Oncol. 2015;33(6):540-9.

49. Zaretsky JM, Garcia-Diaz A, Shin DS, Escuin-Ordinas H, Hugo W, HuLieskovan S, et al. Mutations Associated with Acquired Resistance to PD-1 Blockade in Melanoma. N Engl J Med. 2016;375(9):819-29.

50. Amiot L, Ferrone S, Grosse-Wilde H, Seliger B. Biology of HLA-G in cancer: a candidate molecule for therapeutic intervention? Cell Mol Life Sci. 2011; 68(3):417-31.

51. Siebert JC, Wagner BD, Juarez-Colunga E. Integrating and mining diverse data in human immunological studies. Bioanalysis. 2014;6(2):209-23.

52. Yabu JM, Siebert JC, Maecker HT. Immune Profiles to Predict Response to Desensitization Therapy in Highly HLA-Sensitized Kidney Transplant Candidates. PLoS One. 2016;11(4):e0153355.

53. Rahma OE, Hamilton JM, Wojtowicz M, Dakheel O, Bernstein S, Liewehr DJ, et al. The immunological and clinical effects of mutated ras peptide vaccine in combination with IL-2, GM-CSF, or both in patients with solid tumors. J Transl Med. 2014;12:55.

54. Zdanov S, Mandapathil M, Abu Eid R, Adamson-Fadeyi S, Wilson W, Qian J, et al. Mutant KRAS Conversion of Conventional T Cells into Regulatory $T$ Cells. Cancer Immunol Res. 2016;4(4):354-65.

55. Fridman WH, Pages F, Sautes-Fridman C, Galon J. The immune contexture in human tumours: impact on clinical outcome. Nat Rev Cancer. 2012;12(4):298-306.

56. Becht E, Giraldo NA, Germain C, de Reynies A, Laurent-Puig P, Zucman-Rossi J, et al. Immune Contexture, Immunoscore, and Malignant Cell Molecular Subgroups for Prognostic and Theranostic Classifications of Cancers. Adv Immunol. 2016;130:95-190.

57. Pages F, Kirilovsky A, Mlecnik B, Asslaber M, Tosolini M, Bindea G, et al. In situ cytotoxic and memory $T$ cells predict outcome in patients with earlystage colorectal cancer. J Clin Oncol. 2009;27(35):5944-51.

58. Angelova M, Charoentong P, Hackl H, Fischer ML, Snajder R, Krogsdam AM, et al. Characterization of the immunophenotypes and antigenomes of colorectal cancers reveals distinct tumor escape mechanisms and novel targets for immunotherapy. Genome Biol. 2015;16:64

59. Anitei MG, Zeitoun G, Mlecnik B, Marliot F, Haicheur N, Todosi AM, et al. Prognostic and predictive values of the immunoscore in patients with rectal cancer. Clin Cancer Res. 2014;20(7):1891-9.

60. Gros A, Parkhurst MR, Tran E, Pasetto A, Robbins PF, Ilyas S, et al. Prospective identification of neoantigen-specific lymphocytes in the peripheral blood of melanoma patients. Nat Med. 2016;22(4):433-8.

61. Bendall SC, Nolan GP, Roederer M, Chattopadhyay PK. A deep profiler's guide to cytometry. Trends Immunol. 2012;33(7):323-32.

62. Butler MO, Friedlander P, Milstein MI, Mooney MM, Metzler G, Murray AP, et al. Establishment of antitumor memory in humans using in vitro-educated CD8+ T cells. Sci Transl Med. 2011;3(80):80ra34.

63. Germain C, Gnjatic S, Tamzalit F, Knockaert S, Remark R, Goc J, et al. Presence of $B$ cells in tertiary lymphoid structures is associated with a protective immunity in patients with lung cancer. Am J Respir Crit Care Med. 2014;189(7):832-44.

64. Jager E, Nagata Y, Gnjatic S, Wada H, Stockert E, Karbach J, et al. Monitoring CD8 T cell responses to NY-ESO-1: correlation of humoral and cellular immune responses. Proc Natl Acad Sci U S A. 2000;97(9):4760-5.

65. Gnjatic S, Atanackovic D, Jager E, Matsuo M, Selvakumar A, Altorki NK, et al. Survey of naturally occurring CD4+ T cell responses against NY-ESO-1 in cancer patients: correlation with antibody responses. Proc Natl Acad Sci U S A. 2003;100(15):8862-7.
66. Gure AO, Chua R, Williamson B, Gonen M, Ferrera CA, Gnjatic S, et al. Cancer-testis genes are coordinately expressed and are markers of poor outcome in non-small cell lung cancer. Clin Cancer Res. 2005;11(22): 8055-62.

67. Yuan J, Adamow M, Ginsberg BA, Rasalan TS, Ritter E, Gallardo HF, et al. Integrated NY-ESO-1 antibody and CD8+ T-cell responses correlate with clinical benefit in advanced melanoma patients treated with ipilimumab. Proc Natl Acad Sci U S A. 2011;108(40):16723-8.

68. Lepone LM, Donahue RN, Grenga I, Metenou S, Richards J, Heery CR, et al.: Analyses of 123 Peripheral Human Immune Cell Subsets: Defining Differences with Age and between Healthy Donors and Cancer Patients Not Detected in Analysis of Standard Immune Cell Types. J Circ Biomark 2016, 5. doi: 10.5772/62322.

69. Heery CR, Ibrahim NK, Arlen PM, Mohebtash M, Murray JL, Koenig K, et al. Docetaxel Alone or in Combination With a Therapeutic Cancer Vaccine (PANVAC) in Patients With Metastatic Breast Cancer: A Randomized Clinical Trial. JAMA Oncol. 2015;1(8):1087-95.

70. Heery CR, Singh BH, Rauckhorst M, Marte JL, Donahue RN, Grenga I, et al. Phase I Trial of a Yeast-Based Therapeutic Cancer Vaccine (Gl-6301) Targeting the Transcription Factor Brachyury. Cancer Immunol Res. 2015; 3(11):1248-56.

71. Kudo-Saito C, Schlom J, Hodge JW. Induction of an antigen cascade by diversified subcutaneous/intratumoral vaccination is associated with antitumor responses. Clin Cancer Res. 2005;11(6):2416-26.

72. Disis ML, Wallace DR, Gooley TA, Dang Y, Slota M, Lu H, et al. Concurrent trastuzumab and HER2/neu-specific vaccination in patients with metastatic breast cancer. J Clin Oncol. 2009;27(28):4685-92.

73. Hardwick N, Chain B. Epitope spreading contributes to effective immunotherapy in metastatic melanoma patients. Immunotherapy. 2011;3(6):731-3.

74. Walter S, Weinschenk T, Stenzl A, Zdrojowy R, Pluzanska A, Szczylik C, et al. Multipeptide immune response to cancer vaccine IMA901 after single-dose cyclophosphamide associates with longer patient survival. Nat Med. 2012; 18(8):1254-61.

75. Gulley JL, Madan RA, Tsang KY, Jochems C, Marte JL, Farsaci B, et al. Immune impact induced by PROSTVAC (PSA-TRICOM), a therapeutic vaccine for prostate cancer. Cancer Immunol Res. 2014;2(2):133-41.

\section{Submit your next manuscript to BioMed Central and we will help you at every step:}

- We accept pre-submission inquiries

- Our selector tool helps you to find the most relevant journal

- We provide round the clock customer support

- Convenient online submission

- Thorough peer review

- Inclusion in PubMed and all major indexing services

- Maximum visibility for your research

Submit your manuscript at www.biomedcentral.com/submit
Biomed Central 